\title{
Social Psychological Analysis of College Students' Abnormal Consumption from the Perspective of "Social Identity"
}

\author{
Kerui Zhang \\ College of Letters and Science, University of California, Davis, CA 95616, United States \\ krizhang@ucdavis.edu
}

\begin{abstract}
Among college students, there is excessive consumption in pursuit of material enjoyment. They show strong spending power, which is very inconsistent with their own economic situation in daily life and online social platforms, leading to online shopping overdraft, online lending and "naked lending" and other chaos. The basic meaning of "social identity theory" is that individuals realize that they belong to a specific social group, and that being a group member will bring them emotional and value significance. In view of the present situation of abnormal consumption of college students, this paper explores its influencing factors from the perspective of psychological culture from the perspective of "social identity", emphasizes the important role of psychological behavior of college students' consumption, and then evaluates the rational and moderate development trend of education consumption. Establish a reasonable and moderate concept of educational consumption, and provide valuable theoretical guidance for the cultivation of talents and the healthy, harmonious and sustainable development of society. This paper analyzes the formation of social identity theory, the connotation of consumer identity and the influence mechanism of social identity on consumer behavior, and analyzes the abnormal consumption of contemporary college students from the perspective of social psychology.
\end{abstract}

Keywords: From the Perspective of "Social Identity"; College Students' Abnormal Consumption; Social Psychology.

\section{Introduction}

The healthy growth of teenagers, especially contemporary college students, is related to the future development of the country and the rejuvenation of the Chinese nation. In the group of college students, excessive consumption in pursuit of material enjoyment has emerged. Their daily life and online social platforms show strong spending power which is extremely inconsistent with their own economic situation, leading to chaos such as online shopping overdraft, peer-to-peer lending and "naked lending" [1]. Its characteristics often reflect unreasonable and unscientific abnormal consumption tendency, which will not only affect one's future, but also bring serious harm to parents [2]. This abnormal consumption phenomenon reflects that some college students' self-awareness and consumption concept are immature, which is because they have not established correct values [3]. Therefore, it is of great theoretical and social significance to pay attention to the abnormal consumption of college students, grasp its characteristics and analyze the abnormal consumption from the perspective of social psychology [4]. Therefore, it is of great practical significance to deeply study the abnormal consumption behavior of contemporary college students and provide timely and active guidance.

Looking at China's educational practice from the perspective of social development and personnel training, paying attention to the social identity of educational consumption has become an important issue in China's current educational development [5]. From the current research results and literature, people mainly pay attention to the internal factors that affect self-control in learning, and pay less attention to the influence of environment and group factors on self-control in learning [6]. Understanding and guiding this issue will become an unavoidable social problem in the future development of Chinese education [7]. The basic meaning of "social identity theory" is that an individual realizes that he belongs to a specific social group, and being a member of this group will bring him or her emotional and value significance [8]. As a research sample, the influencing factors of students' learning self-control are too complicated or have no universal research significance, so 
that the control level of irrelevant variables is required to be higher in the research [9]. Paying attention to education consumption and guiding people to establish a reasonable concept of education consumption is of great strategic significance to personnel training and social development. Therefore, the sample selection of regular school groups is not suitable for detailed research on the influencing factors of students' learning self-control at the micro level [9].

\section{The Main Types of Abnormal Consumption Behavior of Contemporary College Students}

\subsection{Comparative Consumption Behavior}

Under normal circumstances, consumers meet their consumption needs according to their own economic income level, but blindly climb up from the actual income level. Due to the strong selfesteem, competitive heart and vanity of college students, it is very easy to breed the abnormal psychology of overwhelming each other and seeking to be the leader in consumption behavior, resulting in the behavior of comparing consumption and showing off consumption. In the social environment, college students' social relations are not limited to their friends [10]. With the advent of the era of globalization, Western fashionable consumption ideas have also had a certain impact on College Students' consumption ideas. When students imitate western consumption ideas, they are easy to cause comparison psychology, and many students agree with the concept of timely enjoyment. Some college students who pursue individuality and novelty and fashion are driven by the psychology of being competitive and indomitable, and often show off some consumer goods in online social circles or friend's gatherings, thus creating a vanity illusion of petty bourgeoisie and unique taste. This phenomenon has increased the economic burden on families, especially poor families, and prevented students from successfully completing their studies, and led some students to go astray. This kind of comparison consumption is the product of individual vanity psychology and competitive desire, which materializes all the comparative factors and tries to fill some disadvantages of consumers' own lack of ability through shopping.

\subsection{Emotional Consumption Behavior}

Emotional consumption refers to consumers' strong emotional response to external stimuli, which leads to unplanned consumption behavior. Most of the college students left their parents for the first time. They didn't have a good opportunity to manage their money and control their money for the first time. They lacked planning and a reasonable concept of consumption. Many students do not make consumption plans for each month or the whole semester in advance. They often "live beyond their means" and pursue impromptu consumption. "Emotional demand" replaces "practical demand" as the main motivation of consumption. Students with better economic conditions often spend irrationally blindly, thus changing their initial stage. There is also a relative "social identity" in the group. For the members in the informal group of students, it can be said that self-control is influenced by social identity, which makes willpower and temptation infectious. The results of correlation analysis between different types of students' informal groups and learning self-control are shown in Table 1.

Table 1. Significant differences in scores of self-controls under different types

\begin{tabular}{|c|c|c|c|c|c|c|}
\hline \multicolumn{7}{|c|}{ Automatic control ANOVA } \\
\hline \multirow{3}{*}{ Self-control } & Sum of squares & Df & Mean square & F & Significance \\
\cline { 2 - 7 } & Between groups & 6.564 & 2 & .556 & 0.785 & .006 \\
\cline { 2 - 7 } & Within group & 66.8 & 258 & .350 & & \\
\cline { 2 - 8 } & Total & 73.415 & 260 & & & \\
\hline
\end{tabular}


College students also like to pursue new, strange and beautiful, and are eager to be recognized by other students or attract others' attention. Emotional consumption, the main way to release emotion and vent themselves, is very easy to make some college students overdraw their consumption ability, so they fall into the dilemma of borrowing from others, and some even fall into the traps of illegal lending and "naked lending" on the network platform. Based on relevant research, the information is coded, with positive influence, intermediate influence, negative influence, gender and grade as independent variables and learning self-control as dependent variable, and the results of stepwise regression analysis are shown in Table 2.

Table 2. Stepwise regression with learning self-control as dependent variable

\begin{tabular}{|c|c|c|c|c|c|c|}
\hline \multicolumn{7}{|c|}{ Coefficient a } \\
\hline Model & $\begin{array}{c}\text { Non-standardized } \\
\text { coefficient }\end{array}$ & $\begin{array}{c}\text { Standard } \\
\text { coefficient }\end{array}$ & $\mathrm{t}$ & Sig. \\
\hline 1 & B & $\begin{array}{c}\text { Standard } \\
\text { error }\end{array}$ & Trial version & & \\
\hline & $\begin{array}{c}\text { Positive } \\
\text { influence form }\end{array}$ & .158 & .114 & & 30.540 & .000 \\
\hline $\begin{array}{c}\text { A. dependent } \\
\text { variable: }\end{array}$ & Self-control & & .272 & .133 & 2.183 & .028 \\
\hline
\end{tabular}

With the arrival of the information age, the information is spreading rapidly. Some media transmit the consumption trend to the campus by producing a large amount of information. The inexperienced college students can't clearly understand that society loses its due judgment ability in consumption, resulting in serious blind consumption.

\subsection{High-end Consumption Behavior}

For the so-called "face", some college students pay attention to taste, famous brand and ostentation, do not consider their own economic ability, buy a large number of High-end goods, do not go to nonHigh-end hotels for dinner, wear famous brands and blindly pursue High-end consumption. At present, students' ideas are relatively open and they like to play tricks in consumption. In order not to repeat the same activities, feel different experiences and allow themselves to participate in high consumption activities, a large number of students are pursuing different consumption patterns. The expanding consumption desire will inevitably lead to unbalanced phenomenon and abnormal consumption. Growing up in the information explosion era, college students have a lot of treasures about big-name luxury goods, as if breaking away from the public opinion circle of luxury goods is an outdated performance. Today, with the rapid development of economy, Chinese people's demand for quality of life and comfort is getting higher and higher, so consumption behavior becomes a trend, and consumerism trend of thought arises at the historic moment. This abnormal consumption not only makes their lives fall into another strange circle, but also has an immeasurable impact on their healthy growth. This fashionable and novel consumption is often not within the scope of students, and they do not think that their consumption concept is wrong. Instead, they become more addicted to this kind of consumption and feel the pleasure brought by this kind of consumption.

\section{The Influence of Social Identity on Community Consumption Behavior}

\subsection{Connotation of Community Marketing.}

Community marketing is a business form based on common value points, forming people's aggregation and marketing carrier through social tools, and providing products or services required by community members. Based on the sociological perspective, consumer sociologists point out that consumption, as a social activity, is a specific way to create identity. Educational consumption is a special social identity behavior. Through educational consumption, people reflect their views, 
positioning, evaluation and recognition of their social role and status. Different from the macro perspective of consumer sociology, marketing scholars have done a lot of research on the influence of consumer's gender, religion, race and occupation identity on consumer behavior from the micro level, and pointed out the existence of consumer identity. The process and mechanism of social identity affecting consumers' attitudes and behaviors can be described by Figure 1 .

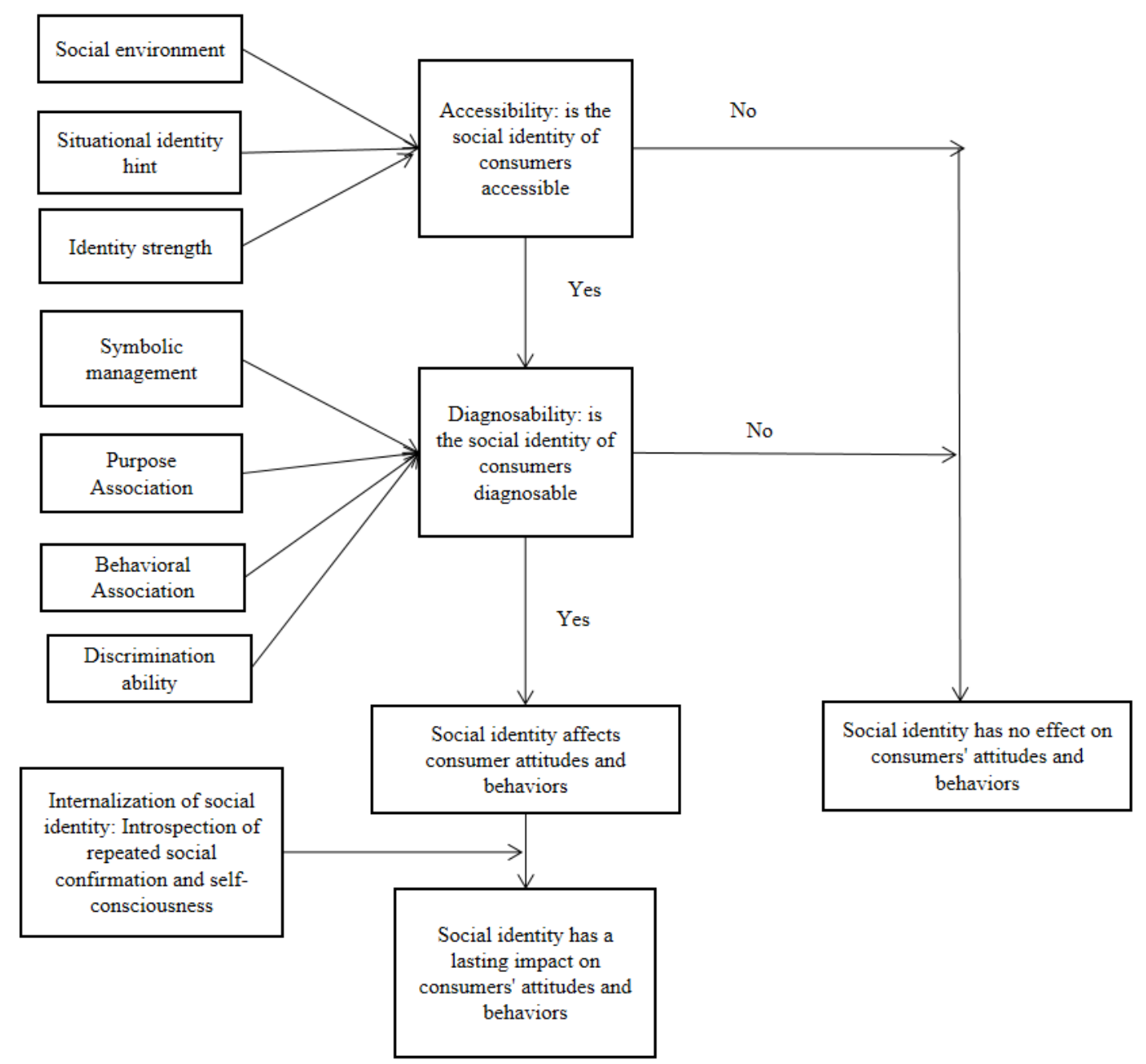

Figure 1. Impact mechanism of social identity on consumer behavior

Because some groups have advantages over other groups in social status, power and resources, they have a sense of group superiority, and there are levels between groups. Online community marketing is not limited by time and space. It can not only rely on WeChat, but also take other online social platforms as carriers. Through the pursuit of educational consumption symbols, people have realized the social identity action of a certain value goal, thus achieving the repositioning of objective identity. The construction and expansion of the mechanism model of social identity influencing consumer behavior by scholars can help researchers clearly understand the process of social identity.

\subsection{The Embodiment of Social Identity in Community Consumption Behavior}

From the cognitive point of view, the group is psychologically treated, by classifying people and getting positive emotions and values, and distinguishing between self and others, so that individuals can make more consistent social behaviors to their psychologically affiliated social groups. Individuals with positive social identity will invest a lot of money and energy in maintaining their identity and defend their image characteristics compared with other groups. With the change of consumption situation, consumers' behavior will have different performances. In this process, 
consumers' self-awareness and social culture have an important impact on consumption decisionmaking behavior. In social comparison, people often use positive labels to define or evaluate inner groups, while negative words to describe outer groups, thus increasing self-esteem. Social identity is based on subjective psychology. It is a subjective feeling and attitude towards self-identity, which is relatively stable and sustainable. The research on consumer behavior from the perspective of social identity attempts to effectively explain the differences in consumer behavior according to consumers' psychological cognition of social identity classification. According to social identity theory, selfesteem and self-motivation are the most basic demand motivation of individual behavior and group behavior. Similarly, people can express their identity or difference with others or social groups through the expression of educational consumption. Try to make the individual realize his identity and qualification in his group, and the value and emotional impact of this identity and qualification on his social perception and social behavior.

\subsection{The Influence of Social Identity on Community Consumption Behavior}

The identity of an individual in the community and the community to which he belongs are an important part of a person's self-concept. Therefore, when people are socializing, they do not face others with individual behavior characteristics, but communicate with others with the common behavior characteristics of a class or group of people. Under the influence of social comparison, some people will actively identify with those groups that are more attractive to themselves, and try their best to change their objective identity and try to join them. Social identity and consumption decisionmaking are the products of specific social situations, which are affected by specific cultural environment. In social communication, when individuals want to compare their opinions or abilities with others, they often make social comparison with groups that are more like the group to which they belong. In order to obtain social recognition and improve self-esteem, people will strive to obtain positive and positive social recognition. Education creates a way to realize the formation, creation, maintenance and management of personal social identity. When individuals get positive results in social comparison and positive differentiation, they will show more inner group preference. In view of different countries and regions, it is of great significance to select social groups and consumers in different cultural backgrounds as the research objects to study the influence of social identity on consumer behavior in specific cultural situations.

\subsection{Experimental Results and Analysis}

In order to verify the role of face view between social identity and product consumption behavior, this study uses the trilogy of Baron and Kenny (1986), as shown in table and Table 3.

Table 3. (Mobile phone, call fee) Intermediary effect analysis

\begin{tabular}{|c|c|c|c|c|c|}
\hline Step & Independent variable & Dependent variable & Standardization coefficient & $\mathrm{T}$ value & Sig \\
\hline Step 1 & Social identity & Product consumption & -0.262 & -4.930 & 0.000 \\
\hline \multirow{3}{*}{ Step 2} & \multirow{3}{*}{ Social identity } & Want face & $0.531 \ldots$ & 11.298 & 0.000 \\
\hline & & Fear of losing face & $0.445 \ldots$ & 8.947 & 0.000 \\
\hline & & View of face & $0.558 \ldots$ & 12.162 & 0.000 \\
\hline \multirow{6}{*}{ Step 3} & Social identity & \multirow{6}{*}{ Product consumption } & -0.143. & -2.322 & 0.020 \\
\hline & Want face & & $-0.225 \ldots$ & -3.646 & 0.000 \\
\hline & Social identity & & -0.128 & -2.243 & 0.024 \\
\hline & Fear of losing face & & $-0.303 .$. & -5.299 & 0.000 \\
\hline & Social identity & & -0.072 & -1.189 & 0.234 \\
\hline & View of face & & -0.339 & -5.512 & 0.000 \\
\hline
\end{tabular}

The results show that social identity has a significant impact on the service consumption of two different products (mobile phone, phone bill) and (clothing, customized service) $(\beta-0.262, \mathrm{P}<0.001$; $\beta=0.322, P<0.001)$, social identity has a significant effect on the desire for face, fear of losing face and face outlook $(\beta=0.531, \mathrm{P}<0.001 ; \beta=0.445, \mathrm{P}<0.001 ; \beta=0.558, \mathrm{P}<0.001)$. Taking "product 
consumption" as the dependent variable and "social identity" and "wanting face" as the independent variables, this paper makes a regression analysis on the combination of the two categories (products and services). It is found that in the influence of (mobile phone, phone bill) combination, the significance of "social identity" is weakened $(\beta=-0.143, \mathrm{P}<0.05)$, and the influence of "wanting face" is significant $(\beta=-0.225, \mathrm{P}<0.001)$. The face view is introduced into the model as a whole, and the regression analysis is carried out with "product consumption" as the independent variable and "social identity" and "face view" as the independent variables. It is found that the "face view" has a significant impact on the two combinations (mobile phone, telephone charge) and (clothing, customization) in "product consumption" $(\beta=-0.339, \mathrm{P}<0.001 ; \beta=0.313, \mathrm{P}<0.001)$, while the impact of "social identity" on the two combinations of "product consumption" is no longer significant ( $\beta=-$ $0.072, \mathrm{P}>0.05 ; \beta=0.147, \mathrm{P}>0.05$ ). It can be seen that after controlling the intermediary variable, the influence of the independent variable on the dependent variable becomes insignificant, indicating that the concept of face plays a complete intermediary role. Therefore, the concept of face is an intermediary variable between group identity and product service consumption, and the intermediary effect is significant, and $\mathrm{H} 3$ is supported.

Table 4. Intermediary effect analysis of clothing and customized services

\begin{tabular}{|c|c|c|c|c|c|}
\hline Step & Independent variable & Dependent variable & Standardization coefficient & T value & Sig \\
\hline Step 1 & Social identity & Product consumption & -0.322 & 6.170 & 0.000 \\
\hline \multirow{3}{*}{ Step 2} & \multirow{3}{*}{ Social identity } & Want face & $0.531 \ldots$ & 11.298 & 0.000 \\
\hline & & Fear of losing face & $0.445 \ldots$ & 8.947 & 0.000 \\
\hline & & View of face & $0.600 \ldots$ & 12.162 & 0.000 \\
\hline \multirow{6}{*}{ Step 3} & Social identity & \multirow{6}{*}{ Product consumption } & -0.203 & 3.356 & 0.002 \\
\hline & Want face & & $-0.225 \ldots$ & 3.725 & 0.000 \\
\hline & Social identity & & $-0.205 \ldots$ & 3.628 & 0.000 \\
\hline & Fear of losing face & & $-0.264 \ldots$ & 4.670 & 0.000 \\
\hline & Social identity & & -0.147 & 1.933 & 0.053 \\
\hline & View of face & & $-0.313 \ldots$ & 5.157 & 0.000 \\
\hline
\end{tabular}

Focusing on the service-oriented consumption of products, combined with the cultural value variable of "face view", this paper tests the influence mechanism of internal group identity on the service-oriented consumption of products, and draws the following conclusions based on effective samples.

(1) Social identity has a significant effect on product consumption. For modern Chinese consumers with different social identities, when they consume (products and services) in combination, they do not focus on the attribute interests of physical products and ignore the service value, but pay differential attention to different types of service elements in (products and services) combination.

(2) Social identity has a significant impact on product consumption through the concept of face. Social identity can stimulate consumers' view of face. The higher the social identity of consumers, the more their behavior is affected by the concept of face; The difference of face view contributes to the difference of product consumption.

\section{Conclusion}

The above analysis shows that college students' abnormal consumption has its profound social and psychological reasons. At present, China is in the era of consumption upgrading. More and more consumer participation behaviors are produced, and they tend to be psychological and spiritual. The continuous development of China's economy and the wave of global economy have brought great changes to college students' consumption concept. To a certain extent, social psychology has led to college students' abnormal consumption concept. We should give full play to the power and role of society, schools, parents and college students themselves. Community is the new normal of individual or enterprise marketing mode in China, and community consumption behavior with high 
psychological identity will be valued by more enterprises. According to the characteristics of college students' consumption psychology, we should take necessary, reasonable and scientific measures to guide them to correctly handle the relationship between consumption, money and energy. With simple life instead of extravagant life, the formation of consumption concept will also make college students have a good sense of social responsibility and establish correct life values.

\section{References}

[1] Taylan S, Zkan L. The Effect of Instructors' Clinical Experience on Professional Socialization from the Perspective of Nursing Students: A Phenomenological Qualitative study[J]. Nurse Education Today, 2021, 99 (1):104797-104799.

[2] Wang Q, Jiang X T, Yang X, et al. Comparative analysis of drivers of energy consumption in China, the USA and India - A perspective from stratified heterogeneity[J]. The Science of the Total Environment, 2020, 698(Jan.1):134117.1-134117.10.

[3] Sustainability. Social Networks Consumption and Addiction in College Students during the COVID-19 Pandemic: Educational Approach to Responsible Use[J]. Sustainability, 2020, 12-14.

[4] EH Hernández, Moreno-Murcia J A, J Espín. Teachers' interpersonal styles and fear of failure from the perspective of physical education students[J]. PLOS ONE, 2020, 15-20.

[5] Polites G L, Serrano C, Thatcher J B, et al. Understanding social networking site (SNS) identity from a dual systems perspective: an investigation of the dark side of SNS use[J]. European journal of information systems, 2018, 27(5):600-621.

[6] Huang Q, Li X, Zhao J, et al. Social Networking Reduces Peak Power Consumption in Smart Grid[J]. IEEE Transactions on Smart Grid, 2017, 6(3):1403-1413.

[7] Tójar-Hurtado Juan-Carlos, Mena-Rodríguez Esther, Fernández-Jiménez Miguel-ángel. Spanish Agriculture and Water: Educational Implications of Water Culture and Consumption from the Farmers' Perspective [J]. Water, 2017, 9(12):964-696.

[8] Marjanovic U, Simeunovic N, Delic M, et al. Assessing the success of university social networking sites: engineering students' perspective [J]. The International journal of engineering education, 2018, 34(4): págs. 1363-1375.

[9] Oh S, Pandey M, Kim I, et al. Image-oriented economic perspective on user behavior in multimedia social forums: An analysis on supply, consumption, and saliency[J]. Pattern Recognition Letters, 2016, 72 (mar.1): 33-40.

[10] Thomas J M, Liu J, Robinson E L, et al. Descriptive and liking social norm messages enhance the consumption of a cruciferous vegetable in healthy students: sustained effects after a 24-hour delay[J]. Appetite, 2016, 101:218-220. 